\title{
Older, Heavier, Arthritic, Psychiatrically Disordered, and Opioid-Familiar Patients Are at Risk for Opioid Use After Medial Patellofemoral Ligament Reconstruction
}

\author{
Joshua P. Castle, M.D., Toufic R. Jildeh, M.D., Patrick J. Buckley, B.S., \\ Muhammad J. Abbas, B.S., Salma Mumuni, M.D., and Kelechi R. Okoroha, M.D.
}

\begin{abstract}
Purpose: To investigate which factors predispose patients for prolonged opioid use after medial patellofemoral ligament (MPFL) reconstruction. Methods: A retrospective review of all patients who underwent MPFL reconstruction at a single institution between January 2013 and June 2020 was conducted. Opioid consumption before and after surgery was recorded and confirmed using Michigan Automated Prescriptions System monitoring program. Patients were classified into preoperative opioid users and nonusers. Risk factors for continued opioid use were assessed by collecting patient demographic variables, psychiatric history, number of previous patellar dislocations, and operative factors. Results: A total of 102 patients were included during the time frame of interest. Patients were on average $21.6 \pm 8.5$ years old with a mean body mass index of $28.2 \pm 7.9$. Thirty patients $(29.0 \%)$ sustained $>10$ dislocations preoperatively. Preoperative opioid use was present in 13 $(12.7 \%)$ patients. Greater than 10 dislocations (odds ratio [OR] 5.00, 95\% confidence interval [CI] 1.12-20.92) and psychiatric history (OR 3.33, 95\% CI, 1.2-9.1; $P=.016$ ) significantly predicted opioid refills the first month after surgery. Risk factors for opioid refills at 2 to 12 months postoperatively included smoking (OR 4.50, 95\% CI 1.13-17.96), preoperative opioid use (OR 7.32, 95\% CI 1.88-28.47), psychiatric disorder (OR 3.77, 95\% CI 2.3-6.2; $P<.001$ ), age $>30$ years (OR 7.03, 95\% CI 3.63-13.61; $P<.001$ ), and obesity (OR 2.68, 95\% CI 1.40-5.14; $P=.002$ ). Compared with Outerbridge 0 , a greater percentage of patients with Outerbridge 1 or 2 and 3 or 4 continued using opioids 2 to 12 months after surgery (OR 3.06, 95\% CI 1.33-7.02; $P=.006$ and OR 2.86, 95\% CI 1.24-6.59; $P=.010$, respectively). Conclusions: For patients undergoing MPFL reconstruction, preoperative opioid use, cartilage damage, age $>30$ years, smoking history, body mass index $>30$, and history of psychiatric disorder were found to be significantly associated with prolonged opioid use after surgery. Postoperative opioid refills in this cohort declined after 1 month. Level of Evidence: Level III, retrospective cohort study.
\end{abstract}

From the Department of Orthopaedic Surgery, Henry Ford Hospital (J.P.C., T.R.J., P.J.B., M.J.B.) and Wayne State University School of Medicine (S.M.) Detroit, Michigan; and Department of Orthopedic Surgery, Mayo Clinic, Minneapolis, Minnesota (K.R.O.), U.S.A.

The authors report the following potential conflicts of interest or sources of funding: K.R.O. reports potential conflicts of interest from the following companies: Arthrex (grant and education), Smith $\theta$ Nephew (education, travel and lodging), Pinnacle (education), Medwest Associates (education), Wright Medical Technology (travel and lodging), and Stryker (travel and lodging). Full ICMJE author disclosure forms are available for this article online, as supplementary material.

Primary institution at which the work was performed: Henry Ford Hospital, Detroit, Michigan, U.S.A.

Received April 3, 2021; accepted October 6, 2021.

Address correspondence to Joshua P. Castle, M.D., Department of Orthopaedic Surgery, Henry Ford Hospital, 2799 W. Grand Blvd., Detroit, MI 48202.E-mail: jcastle1@hfhs.org

(C) 2021 THE AUTHORS. Published by Elsevier Inc. on behalf of the Arthroscopy Association of North America. This is an open access article under the CC BY-NC-ND license (http://creativecommons.org/licenses/by-nc-nd/4.0/). 2666-061X/21473

https://doi.org/10.1016/j.asmr.2021.10.012
D espite national recognition and efforts to curtail the opioid epidemic in the United States, opioidrelated deaths continue to rise. From 2000 to 2017, unintentional opioid-related deaths per 100,000 persons climbed from 2.20 to $13.21 .^{1}$ Although the causes of the opioid crisis are multifactorial, opioids prescriptions following surgery are a significant driver of new prescriptions and persistent usage. ${ }^{2}$ Across surgical specialties, orthopaedic surgeons are among the greatest prescribers of opioids postoperatively. ${ }^{3-5}$ Scully et al. $^{4}$ found that orthopaedic surgeons prescribed longer durations of opioids initially and had the greatest refill rates compared with other surgical specialties. These pain medications often go unused ${ }^{6,7}$ and may circulate in the community. ${ }^{6-8}$ In fact, more than onehalf of the states in the United States have instituted legislation to limit opioid prescriptions, which has resulted in significant declines in morphine milligram 
equivalents after orthopaedic procedures. ${ }^{9}$ Multiple studies have investigated risk factors leading to prolonged opioid use after common orthopaedic surgeries (e.g., arthritis, psych history, etc.). ${ }^{7,10-26}$ Identifying predictive factors for prolonged opioid use is crucial for orthopaedic surgeons to tailor pain control for specific patients and practice opioid stewardship.

Although many studies have explored risk factors for opioid consumption after common procedures of the knee, such as arthroscopic meniscus debridement/ repair, anterior cruciate ligament reconstruction, and total knee arthroplasty, there are few studies examining pain control after patellar instability surgery. ${ }^{10}$ Patellar instability includes patella dislocations and subluxations, which commonly occur in young and active individuals. These instability events lead to significant pain, disability, osteochondral defects, and patellofemoral osteoarthritis. ${ }^{27,28}$ Previous literature has demonstrated up to a $49 \%$ risk of recurrent patellar instability in those with a previous history of instability $^{29}$ and often require surgery such as medial patellofemoral ligament (MPFL) reconstruction.

The purpose of this study was to investigate which factors predispose patients to prolonged opioid use after MPFL reconstructing. Based on previous literature evaluating risk factors for prolong opioid use following operative management, we hypothesized that preoperative opioid use, psychiatric diagnosis history, and osteoarthritis would be significantly associated with prolonged opioid consumption.

\section{Methods}

The Strengthening the Reporting of Observational Studies in Epidemiology (STROBE) guidelines for reporting observational studies were followed during the completion of this study. Our institutional review board approved the study before study initiation and data collection. Institutional review board approval was obtained for this project from the primary institution, Henry Ford Hospital. A retrospective review of all patients who underwent MPFL reconstruction or repair from August 2013 to July 2020 at a single institution across 6 fellowship-trained sport surgeons was conducted. Recruitment period was determined based on availability of patient information in electronic medical record. Participants were identified using Current Procedural Terminology codes 27422, 27427, and 27428. Patients with documented complications, such as infections, blood clots, patellofemoral arthrosis, and reinjury, separate injuries in the 12 -month postoperative period, and opioids prescribed for conditions unrelated to the operative condition were excluded from the study. Using a previously verified study methodology, ${ }^{10,11}$ we further categorized participants into 2 groups based on opioid consumption. Preoperative opioid users were classified as those receiving opioid prescriptions within 3 months before surgery whereas nonusers as those without any prescriptions within 3 months of surgery. Opioid prescriptions were identified through chart review and were confirmed by the statewide prescription monitoring program (Michigan Automated Prescriptions System) for controlled substance (schedule 2-5 drugs).

Postoperative opioid prescriptions from either the primary surgeon or other providers were monitored for 12 months after surgery following MPFL reconstruction. The number of the prescription refills and date after surgery were recorded. Information on demographics such as age, sex, body mass index (BMI), race, psychiatric history, smoking, and workman's compensation were collected. Surgeon notes were queried for history of recurrent dislocation, operative factors such as cartilage damage (Outterbridge classification and location of cartilage damage), types of grafts used (autograft vs allograft), number of anchors, and concomitant procedures performed. ${ }^{30}$

\section{Data Analysis}

Continuous variables were reported as means and standard deviations, whereas categorial variables were reported as counts and column percentages. Univariate 2 -group comparisons using Wilcoxon rank sum tests were conducted for continuous variables and $\chi^{2}$ or Fisher exact tests for categorial variables. Distributions were compared between more than 2-level groups using the Kruskal-Wallis test. The relation between continuous variables was examined using a Spearman correlation. Statistical significance was set at $P<.05$. All analyses were performed using SAS software (version 9.4; SAS Institute, Cary, NC).

\section{Results}

A total of 203 patients were identified using Current Procedural Terminology, and 101 met exclusion criteria or lacked required data points for study inclusion. A total of 102 patients who underwent MPFL reconstruction at a single institution were included. Patients in the cohort were on average $21.6 \pm 8.5$ years old (range $11-53$ years) and had a BMI of $28.2 \pm 7.9$ (range 16.3-56.4). Thirteen patients $(12.7 \%)$ consumed opioids within the 3 months before surgery, whereas 89 $(87.3 \%)$ patients were classified as nonusers. Demographic variables, procedures performed, history of previous dislocations, concomitant surgery, and Outerbridge classifications are displayed in Table 1.

\section{Opioid Use Postoperatively}

In the first month following surgery, 33 patients $(32.4 \%)$ obtained an opioid prescription refill. In months 2 to 12 postoperatively, 14 patients $(13.7 \%)$ received a refill. Only 4 patients $(3.9 \%)$ received more 
Table 1. Demographics

\begin{tabular}{lr}
\hline Total patients, $\mathrm{n}$ & 102 \\
Patients with an opioid script before surgery & $13(12.7 \%)$ \\
Patients with concomitant surgery & $100(98.0 \%)$ \\
Arthroscopy & $93(91.2 \%)$ \\
Chondroplasty & $42(41.2 \%)$ \\
Synovectomy & $18(17.6 \%)$ \\
Tibial tubercle transfer & $14(13.7 \%)$ \\
Loose body removal & $24(23.5 \%)$ \\
Other & $15(14.7 \%)$ \\
Age, y, mean \pm SD & $21.6 \pm 8.5$ \\
Body mass index, mean \pm SD & $28.2 \pm 7.9$ \\
Age $>30$ years old, & $14(13.7 \%)$ \\
Body mass index $>30$ & $33(32.4 \%)$ \\
Smokers & $13(13 \%)$ \\
10+ dislocations & $30(29 \%)$ \\
Sex & \\
Male & $43(42.2 \%)$ \\
Female & $59(57.8 \%)$ \\
Ethnicity & \\
White & $65(63.7 \%)$ \\
Black & $22(21.6 \%)$ \\
Hispanic & $3(2.9 \%)$ \\
Asian & $3(2.9 \%)$ \\
Unknown & $9(8.8 \%)$ \\
Outerbridge classification & \\
Grade 0 & $24(23.5 \%)$ \\
Grade 1 or 2 & $39(38.2 \%)$ \\
Grade 3 or 4 & $39(38.2 \%)$ \\
\hline SD stand &
\end{tabular}

SD, standard deviation.

than 2 refills in the first year following surgery. Opioid prescription refills are displayed in Figure 1.

\section{Factors Affecting Duration of Opioid Use Postoperatively}

When we evaluated factors that influenced opioid refills in the first month, patients who reported 10 or more patellar dislocations (odds ratio [OR] 5.00, 95\% confidence interval [CI] 1.12-20.92; $P=.028$ ) and those with a history of a psychiatric disorder (including major depressive, generalized anxiety, bipolar, and/or post-traumatic stress disorders) (OR 3.33, 95\% CI 1.21-9.13; $P=.016)$ experienced an elevated risk of opioid refills (Table 2). When we evaluated these same factors for long-term opioid usage 2 to 12 months postoperatively, patients who used opioids preoperatively (OR 7.32, 95\% CI 1.88-28.47; $P<.001$ ), smoked (OR 4.50, 95\% CI 1.13-17.96; $P=.033$ ), had a history of psychiatric disorder (OR 3.77, 95\% CI 2.30-6.17; $P$ $<.001$ ), were older than 30 years old (OR 7.03, 95\% CI 3.63-13.61; $P<.001)$, or had a BMI greater than 30 at the time of surgery (OR 2.68, 95\% CI 1.40-5.14; $P=.002)$ were more likely to refill a narcotic prescription (Table 3). After we stratified patients by opioid use, preoperative opioid users consistently required narcotic refills compared with opioid-naïve patients at all time points beyond 1 month postoperatively (Table 4; Fig 1; $P<.01$ ). No significant
Percentage of Patients Obtaining Opioid Refills (Pre-Op Users vs Opioid Naïve Patients)

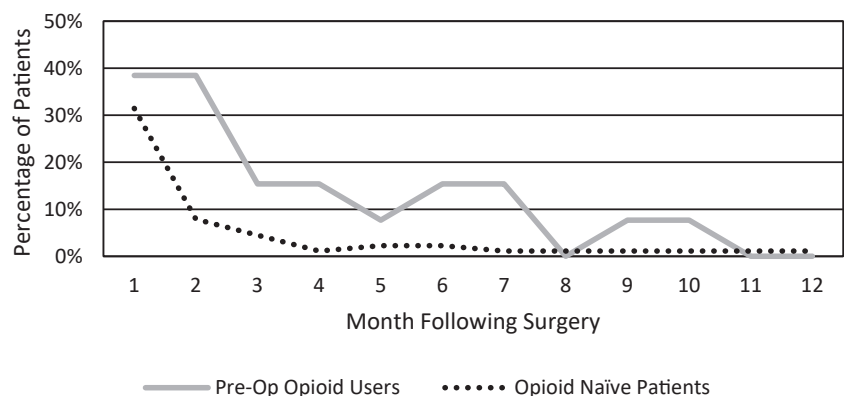

Fig 1. Graph depicting number of patients receiving opioid prescription refills stratified by preoperative opioid use.

differences were found in the number of subsequent prescriptions filled when comparing ethnicity, location of cartilage damage, presence or absence of an avulsion, allograft versus autograft, and number of anchors used.

\section{Outerbridge Classification}

Patients with an intraoperative Outerbridge grade of 1 or 2 (OR 3.06, 95\% CI 1.3-7.0; $P=.006$ ) and 3 or 4 (OR 2.86, 95\%CI 1.2-6.6; $P=.010$ ) were found to consistently require opioid prescriptions beyond 1 month postoperatively compared with patients with an Outerbridge grade 0 (Table 5). However, there was no statistically significant difference between Outerbridge 1 or 2 when compared to Outerbridge 3 or 4 at 1 month postoperatively $(P=.666)$ and $2-12$ months postoperatively $(P=.436)$.

\section{Discussion}

Significant predictors of opioid consumption in the first month post-operatively included 10 or more patellar dislocations and a history of a psychiatric diagnosis. Preoperative opioid consumption does not

Table 2. Opioid Refills, First Month

\begin{tabular}{|c|c|c|c|}
\hline Risk Factor & $\begin{array}{l}\text { Odds } \\
\text { Ratio }\end{array}$ & $\begin{array}{c}95 \% \\
\text { Confidence } \\
\text { Interval }\end{array}$ & $P$ Value \\
\hline Preoperative opioid prescription & 1.36 & $0.41-4.54$ & .615 \\
\hline Smoking & 1.97 & $0.61-6.41$ & .261 \\
\hline 10+ dislocations & 5.00 & $1.12-20.92$ & $.028^{*}$ \\
\hline Psychiatric diagnosis $^{\dagger}$ & 3.33 & $1.21-9.13$ & $.016^{*}$ \\
\hline Age $>30$ years old & 2.38 & $0.76-7.48$ & .129 \\
\hline Body mass index $>30$ & 2.36 & $0.99-5.64$ & .050 \\
\hline Ethnicity & 0.56 & $0.24-1.32$ & .185 \\
\hline Workman's compensation & 1.23 & $0.22-6.71$ & .811 \\
\hline Allograft vs autograft & 1.15 & $0.10-13.21$ & .912 \\
\hline
\end{tabular}

*Significant at $P=.05$

${ }^{\dagger}$ Psychiatric diagnosis includes major depressive disorder, generalized anxiety disorder, bipolar disorder, and post-traumatic stress disorder. 
Table 3. Opioid Long-Term Refill (2-12 Months' Postoperative)

\begin{tabular}{lccc}
\hline \multicolumn{1}{c}{ Risk Factor } & \multicolumn{3}{c}{$95 \%$} \\
& Odds & Confidence \\
& Ratio & Interval & $P$ Value \\
\hline Preoperative opioid prescription & 7.32 & $1.88-28.47$ & $.004^{*}$ \\
Smoking & 4.50 & $1.13-17.96$ & $.033^{*}$ \\
10+ dislocations & 4.25 & $0.47-38.60$ & .200 \\
Psychiatric diagnosis ${ }^{\dagger}$ & 3.77 & $2.30-6.17$ & $<.001^{*}$ \\
Age $>30$ years old & 7.03 & $3.63-13.61$ & $<.001^{*}$ \\
Body mass index $>30$ & 2.68 & $1.40-5.14$ & $.002^{*}$ \\
Ethnicity & 0.36 & $0.11-1.22$ & .100 \\
Workman's compensation & 0.38 & $0.09-1.57$ & .165 \\
Allograft vs autograft & 4.45 & $0.37-54.01$ & .242 \\
\hline$*$ Significant at $P$. & & &
\end{tabular}

*Significant at $P=.05$.

${ }^{\dagger}$ Psychiatric diagnosis includes major depressive disorder, generalized anxiety disorder, bipolar disorder, and post-traumatic stress disorder.

Table 4. Odds Ratio for Opioid Prescription Refill (Compared Preoperative Opioid Users With Opioid-Naïve Patients)

\begin{tabular}{lccc}
\hline & Odds Ratio & $95 \%$ Confidence Interval & $P$ Value \\
\hline First month & 1.36 & $0.41-4.54$ & .614 \\
Months 2-3 & 5.59 & $1.72-16.1$ & $<.001^{*}$ \\
Months 4-6 & 7.71 & $2.12-28.00$ & $<.001^{*}$ \\
Months 7-12 & 4.76 & $1.31-17.25$ & $.009^{*}$ \\
\hline
\end{tabular}

*Statistically significant at $P=.05$.

significantly impact narcotic use in the first postoperative month. At 2 to 12 months postoperatively, preoperative opioid use, smoking, age older than 30 years, a BMI greater than 30, and a history of psychiatric diagnosis significantly predicted opioid consumption. Outerbridge grades 1 or 2 and 3 or 4 significantly predicted opioid use at all time points after surgery. Overall, opioid consumption declined after 2 months and remained relatively low for those undergoing MPFL reconstruction.

Although a minority of patients consumed opioids before surgery, our results indicate that preoperative opioid usage significantly increased the risk for opioid consumption at all time points between 2 and 12 months after surgery (OR 5.48, 95\% CI 2.8-10.7; $P<.001)$. However, it should be noted that the use of narcotics preoperative did not have a significant impact on narcotic use in the first postoperative month. This is likely attributed to the need for adequate postoperative pain control, as more than one-third of both opioid naïve and opioid users required a refill in the first month postoperatively. Across multiple subspecialties in orthopaedic surgery, the literature has consistently demonstrated that preoperative opioid use strongly predicts prolonged opioid demand after surgery. ${ }^{7,10-21,25,26}$ Anthony et al. ${ }^{19}$ conducted a multicenter review of 4,946 ACL reconstructions and found that patients with preoperative prescriptions were 4.06 times (95\% CI, relative risk 3.33-4.96) more likely to be refilling prescriptions at 2 months and 5.35 to 7.67 times more likely at all time points between 3 and 12 months after surgery. The majority of patients discontinued opioid use after the first postoperative month, with $10 \%$ or less refilling at 2 months and beyond. Jildeh et al. ${ }^{10}$ retrospectively examined 735 patients undergoing arthroscopic meniscal surgery. They reported preoperative opioid usage $(P<.001)$ and degree of knee osteoarthritis $(P<.05)$ had a significant effect on postoperative opioid usage. Khazi et al. ${ }^{22}$ retrospectively examined 60 patients undergoing patellofemoral stabilization procedures including MPFL reconstruction $(36.7 \%)$, Fulkerson osteotomy, or both over a 4-year period. Postoperative opioid use precipitously declined from $61.7 \%$ at 2 weeks to $33.3 \%$ at 6 weeks. Similar to our study's results, preoperative opioid consumption independently predicted sustained opioid use at both $2(P=.0023)$ and 6 weeks $(P$ $<.0001)$ compared with the opioid-naïve group. ${ }^{22}$ Most interestingly, the preoperative opioid group experienced worse patient-reported outcome scores (Knee Injury and Osteoarthritis Outcome Score and Kujala) at baseline, 6 months, and 2 years after surgery. Regardless of opioid consumption before surgery, sustained opioid demand at 6 weeks conferred worse patient-reported outcomes at 6 months and 2-year follow-ups. The authors' findings, in conjunction with our results, emphasize the strong predictive value of preoperative opioid use on sustained use after surgery. While this phenomenon is multifactorial, these patients likely develop opioid tolerance and have higher pain requirements. More importantly, Khazi and colleagues'

Table 5. Effect of Outerbridge Classification on Postoperative Opioid Prescription Refills

\begin{tabular}{|c|c|c|c|c|c|c|}
\hline & $\begin{array}{l}\text { First Month } \\
\text { Refills, n (\%) }\end{array}$ & $\begin{array}{c}\text { First Month vs Grade } \\
0 \text { Odds Ratio ( } 95 \% \\
\text { Confidence Interval) }\end{array}$ & $\begin{array}{l}P \text { Value, First } \\
\text { Month vs } \\
\text { Grade } 0\end{array}$ & $\begin{array}{l}\text { Long-Term Refills, } \\
\text { N }(\%)\end{array}$ & $\begin{array}{l}\text { Long-Term vs Grade } \\
0 \text { Odds Ratio (95\% } \\
\text { Confidence } \\
\text { Interval) }\end{array}$ & $\begin{array}{l}\text { P Value Long- } \\
\text { Term vs Grade } 0\end{array}$ \\
\hline Outerbridge Grade 0 & $6(25.0 \%)$ & - & - & $7(2.7 \%)$ & - & - \\
\hline Outerbridge Grade 1 or 2 & $13(33.3 \%)$ & $1.50(0.48-4.68)$ & .484 & $33(7.7 \%)$ & $3.06(1.33-7.02)$ & $.006^{*}$ \\
\hline Outerbridge Grade 3 or 4 & $14(35.9 \%)$ & $1.68(0.54-5.21)$ & .367 & $31(7.2 \%)$ & $2.86(1.24-6.59)$ & $.010 *$ \\
\hline
\end{tabular}

*Statistically significant at $P=.05$. 
results further contribute to the growing evidence that sustained opioid consumption after surgery leads to poorer outcomes. ${ }^{20,23,24}$

Patellofemoral instability commonly leads to osteochondral damage and serves as a catalyst for cartilage deterioration and osteroarthritis. ${ }^{27,31,32}$ Interestingly, our study identified that recurrent instability with greater than 10 patellar dislocations lead to a 5 times significantly increased risk of opioid refills in the first month and a 4.25 times increased risk, although not significant, 2 to 12 months after surgery. Recurrent instability likely caused additional cartilage damage and portended higher pain requirements after surgery. Even after a single traumatic dislocation, Salonen et al. $^{27}$ reported patellofemoral cartilage injury on magnetic resonance imaging in 14 of $20(70 \%)$ of firsttime dislocations, and within 8 years all patients revealed cartilage deterioration. These episodes of instability render patients vulnerable to cartilage damage and eventual long-term osteoarthritic changes. We, also, found that compared with Outerbridge 0, patients with cartilage deterioration, defined as Outerbridge 1 or 2 and 3 or 4 , demonstrated an increased likelihood for opioid refills at all time points after MPFL reconstruction. Previous studies have similarly shown that preoperative knee osteoarthritis predicts prolonged opioid consumption in multiple arthroscopic procedures of the knee such as meniscectomy. ${ }^{10,16,21}$ Surgeons should, however, exhibit caution in patient selection, as significant osteoarthritis at the time of surgery may confer opioid dependence and worse outcomes.

As prescribers, we would be remiss to not acknowledge the role substance use and psychiatric disorders factor into sustained opioid use. Hastings et al. ${ }^{33}$ developed a machine-learning algorithm to predict which patients are at increased risk of adverse opioidrelated outcomes such as dependence, abuse, or poisoning. Previous substance use was among the top predictors for adverse opioid-related outcomes, which included previous benzodiazepines, muscle relaxants, opioids, alcohol, cannabis, and cocaine use. LavoieGagne and colleagues ${ }^{25}$ performed a systematic review of 45 studies including 458,993 patients across various orthopaedic subspecialties to identify risk factors for prolonged opioid use after surgery. Across all studies, previous substance abuse aside from tobacco and alcohol, use of other medications, psychiatric comorbidities, BMI greater than 40, chronic pain conditions, fibromyalgia, and migraines were all associated with prolonged opioid use after surgery. When they examined sports procedures specifically, all previous substance abuse except alcohol abuse was predictive of prolonged opioid consumption. Our results found that psychiatric disorders led to an elevated risk at all time points and smoking increasing the likelihood of postoperative opioid use at 2 to 12 months (OR 4.50, 95\%
CI 1.13-17.96; $P=.033$ ). Previous literature has suggested that patients with a history of psychiatric diagnosis may have altered chemical signaling pathways, which may explain the increased risk of opioid misuse. ${ }^{34}$ While we cannot specifically comment on which psychiatric diagnoses are most vulnerable to opioid dependence, it is crucial for prescribers to holistically evaluate patients for these risk factors and potentially identify patients at greater risk for continued opioid use.

Although $32.4 \%$ of patients refilled an opioid prescription in the first month, only $13.7 \%$ of patients received any refill between months 2 and 12 postoperatively. This can partially be attributed to the success rates and patient population undergoing MPFL reconstruction. Patients undergoing MPFL reconstruction are typically young (average age 24.4 years old) and the majority return to a level of high activity (84.1\% returned to sports) following the surgery. ${ }^{35}$ Baron et al. $^{26}$ retrospectively reviewed a database of 1,316 patients undergoing MPFL reconstruction or tibial tubercle osteotomy and found that age younger than 30 years old, independently predicted lowered opioid use at both 3 and 12 months postoperatively. In addition, chronic preoperative opioid use, obesity (BMI $>30$ ), and a diagnosis of depression or anxiety were all independent risk factors for opioid use at 12 months after surgery. Our results corroborate their findings with those less than 30 years old refilling fewer prescriptions and those with BMI values greater 30 and history of psychiatric diagnosis requiring more refills at 2 to 12 months postoperatively. Patients undergoing patellar stabilization procedures are often younger and likely have a greater baseline activity level. While these younger cohorts are likely to perform well after surgery with lower opioid requirements, more attention should be directed to managing pain in those patients who are older, have more obesity, have history of a psychiatric diagnosis, and the presence of cartilage damage at the time of surgery.

\section{Limitations}

The present study is subject to multiple limitations. The retrospective design of the study depends on the accuracy of the electronic medical record in reporting opioid prescriptions tied to the index procedure. We attempted to mitigate this issue by confirming all prescriptions using the Michigan Automated Prescriptions System monitoring system. Prescriptions unrelated to the procedure were excluded; however, prescription may have been unnecessarily excluded if providers inaccurately associated the indicated diagnoses. In addition, we were unable to determine the exact number of pills consumed from the electronic medical record. Rather, we used opioid refills as a proxy for opioid consumption, a method validated by previous 
studies. ${ }^{10,11}$ The present study demonstrated that perioperative opioid use was relatively low in this group. It is possible that our limited number of preoperative users and small sample size limited our ability to detect significant differences in use in the first month postoperatively. Furthermore, patients were evaluated with greater than 10 dislocations since they would unequivocally represent incompetent MPFL and increased cartilage damaged that has been shown to increase opioid consumption. ${ }^{10}$ Additionally, it was not possible to account for several confounding factors that could have influence postoperative pain and analgesia required. These factors included complexity of surgery, prescriber prescription habits, surgical techniques and rehabilitation protocol, type of anesthesia used, use of nonprescribed narcotics, and the use of non-opioid analgesia. In general, patients undergoing MPFL reconstruction were young, with low BMI values, and were likely healthier individuals, similar to the demographic characteristics of Khazi et al. ${ }^{22}$ These characteristics limit generatability to patients who are older and more overweight.

\section{Conclusions}

For patients undergoing MPFL reconstruction, preoperative opioid use, cartilage damage, age $>30$ years, smoking history, BMI $>30$, and history of psychiatric disorder were found to be significantly associated with prolonged opioid use after surgery. Postoperative opioid refills in this cohort declined after 1 month.

\section{References}

1. Olfson M, Rossen LM, Wall MM, Houry D, Blanco C. Trends in intentional and unintentional opioid overdose deaths in the United States, 2000-2017. JAMA 2019;322: 2340-2342.

2. Hah JM, Bateman BT, Ratliff J, Curtin C, Sun E. Chronic opioid use after surgery: Implications for perioperative management in the face of the opioid epidemic. Anesth Analg 2017;125:1733-1740.

3. Schoenfeld AJ, Jiang W, Chaudhary MA, Scully RE, Koehlmoos T, Haider AH. Sustained prescription opioid use among previously opioid-naive patients insured through TRICARE (2006-2014). JAMA Surg 2017;152: 1175-1176.

4. Scully RE, Schoenfeld AJ, Jiang W, et al. Defining optimal length of opioid pain medication prescription after common surgical procedures. JAMA Surg 2018;153:37-43.

5. Volkow ND, McLellan TA, Cotto JH, Karithanom M, Weiss SR. Characteristics of opioid prescriptions in 2009. JAMA 2011;305:1299-1301.

6. Sabatino MJ, Kunkel ST, Ramkumar DB, Keeney BJ, Jevsevar DS. Excess opioid medication and variation in prescribing patterns following common orthopaedic procedures. J Bone Joint Surg Am 2018;100:180-188.

7. Tepolt FA, Bido J, Burgess S, Micheli LJ, Kocher MS. Opioid overprescription after knee arthroscopy and related surgery in adolescents and young adults. Arthroscopy 2018;34:3236-3243.

8. Sheth U, Mehta M, Huyke F, Terry MA, Tjong VK. Opioid use after common sports medicine procedures: A systematic review. Sports Health 2020;12:225-233.

9. Reid DBC, Shah KN, Shapiro BH, Ruddell JH, Akelman E, Daniels AH. Mandatory prescription limits and opioid utilization following orthopaedic surgery. J Bone Joint Surg Am 2019;101:e43.

10. Jildeh TR, Taylor KA, Khalil LS, et al. Risk factors for postoperative opioid use in arthroscopic meniscal surgery. Arthroscopy 2019;35:575-580.

11. Jildeh TR, Taylor KA, Tramer JS, et al. Risk factors for postoperative opioid use in arthroscopic shoulder labral surgery. Arthroscopy 2020;36:1813-1820.

12. Rao AG, Chan PH, Prentice HA, Paxton EW, Funahashi TT, Maletis GB. Risk factors for opioid use after anterior cruciate ligament reconstruction. Am J Sports Med 2019;47:2130-2137.

13. Namba RS, Singh A, Paxton EW, Inacio MCS. Patient factors associated with prolonged postoperative opioid use after total knee arthroplasty. J Arthroplasty 2018;33: 2449-2454.

14. DeMik DE, Rojas EO, Anthony CA, et al. Opioid prescription refills after osteochondral procedures of the knee. Arthroscopy 2019;35:2083-2088.

15. Rozet I, Nishio I, Robbertze R, Rotter D, Chansky H, Hernandez AV. Prolonged opioid use after knee arthroscopy in military veterans. Anesth Analg 2014;1 19:454-459.

16. Khazi ZM, Baron J, Shamrock A, et al. Preoperative opioid usage, male sex, and preexisting knee osteoarthritis impacts opioid refills after isolated arthroscopic meniscectomy: A population-based study. Arthroscopy 2020;36:2478-2485.

17. Ryan R, Christopher K, Aditya Y, et al. Preparative opioid use is associated with persistent use, readmission and postoperative complications after arthroscopic knee surgery. Arthroscopy 2021;37:1567-1572.

18. Westermann RW, Anthony CA, Bedard N, et al. Opioid consumption after rotator cuff repair. Arthroscopy 2017;33: 1467-1472.

19. Anthony CA, Westermann RW, Bedard N, et al. Opioid demand before and after anterior cruciate ligament reconstruction. Am J Sports Med 2017;45:3098-3103.

20. Forlenza EM, Lavoie-Gagne O, Lu Y, et al. Preoperative opioid use predicts prolonged postoperative opioid use and inferior patient outcomes following anterior cruciate ligament reconstruction. Arthroscopy 2020;36:2681-2688 e2681.

21. Steiner SRH, Cancienne JM, Werner BC. Narcotics and knee arthroscopy: Trends in use and factors associated with prolonged use and postoperative complications. Arthroscopy 2018;34:1931-1939.

22. Khazi ZM, Shamrock AG, Hajewski C, et al. Preoperative opioid use is associated with inferior outcomes after patellofemoral stabilization surgery. Knee Surg Sports Traumatol Arthrosc 2020;28:599-605.

23. Westermann RW, Hu J, Hagen MS, Willey M, Lynch TS, Rosneck J. Epidemiology and detrimental impact of opioid use in patients undergoing arthroscopic treatment of femoroacetabular impingement syndrome. Arthroscopy 2018;34:2832-2836. 
24. Hernandez NM, Parry JA, Mabry TM, Taunton MJ. Patients at risk: Preoperative opioid use affects opioid prescribing, refills, and outcomes after total knee arthroplasty. J Arthroplasty 2018;33:S142-S146.

25. Lavoie-Gagne O, Nwachukwu BU, Allen AA, Leroux T, Lu Y, Forsythe B. Factors predictive of prolonged postoperative narcotic usage following orthopaedic surgery. JBJS Rev 2020;8:e0154.

26. Baron JE, Khazi ZM, Duchman KR, Westermann RW. Risk factors for opioid use after patellofemoral stabilization surgery: A population-based study of 1,316 cases. Iowa Orthop J 2020;40:37-45.

27. Salonen EE, Magga T, Sillanpaa PJ, Kiekara T, Maenpaa H, Mattila VM. Traumatic patellar dislocation and cartilage injury: A follow-up study of long-term cartilage deterioration. Am J Sports Med 2017;45: 1376-1382.

28. Arendt EA, Fithian DC, Cohen E. Current concepts of lateral patella dislocation. Clin Sports Med 2002;21:499-519.

29. Fithian DC, Paxton EW, Stone ML, et al. Epidemiology and natural history of acute patellar dislocation. Am J Sports Med 2004;32:1114-1121.
30. Slattery C, Kweon CY. Classifications in brief: Outerbridge Classification of chondral lesions. Clin Orthop Relat Res 2018;476:2101-2104.

31. Nomura E, Inoue M. Cartilage lesions of the patella in recurrent patellar dislocation. Am J Sports Med 2004;32: 498-502.

32. Sillanpaa PJ, Mattila VM, Visuri $T$, Maenpaa $H$, Pihlajamaki H. Patellofemoral osteoarthritis in patients with operative treatment for patellar dislocation: A magnetic resonance-based analysis. Knee Surg Sports Traumatol Arthrosc 201 1;19:230-235.

33. Hastings JS, Howison M, Inman SE. Predicting high-risk opioid prescriptions before they are given. Proc Natl Acad Sci U S A 2020;117:1917-1923.

34. MacLean RR, Armstrong JL, Sofuoglu M. Stress and opioid use disorder: A systematic review. Addict Behav 2019;98.

35. Schneider DK, Grawe B, Magnussen RA, et al. Outcomes after isolated medial patellofemoral ligament reconstruction for the treatment of recurrent lateral patellar dislocations: A systematic review and meta-analysis. Am J Sports Med 2016;44:2993-3005. 